\title{
Mental animation in the visuospatial sketchpad: Evidence from dual-task studies
}

\author{
VALERIE K. SIMS and MARY HEGARTY \\ University of California, Santa Barbara, California
}

\begin{abstract}
We used the dual-task paradigm to provide evidence that inferring the motion of a component of a mechanical system (mental animation) is a spatial visualization process. In two experiments, participants were asked to solve mental animation problems while simultaneously retaining either a visuospatial working memory load (a configuration of dots in a grid) or a verbal memory load (a list of letters). Both experiments showed that mental animation interferes more with memory for a concurrent visuospatial load than with memory for a verbal load. Experiment 1 also showed that a visuospatial working memory load interferes more with mental animation than does a verbal memory load. Furthermore, Experiment 2 showed that mental animation interferes more with a visuospatial memory load than does a verbal reasoning task that takes approximately the same amount of time.
\end{abstract}

It has been reported that when the inventor Tesla thought of a design for a new device, he first ran a mental simulation of the device for several weeks to see which parts were most subject to wear (O'Neill, 1944, cited in Shepard, 1978). This account is anecdotal, but more systematic analyses of the notebooks of creative scientists suggest that visuospatial imagery plays an important role in problem-solving tasks such as scientific discovery and invention (E. S. Ferguson, 1977; Miller, 1984; Nersessian, 1992). In addition, some more practical activities, such as machine assembly and troubleshooting, have been characterized as involving "envisioning," "running a mental model," or "simulating the behavior of a system in the mind's eye" (DeKleer \& Brown, 1984; Forbus, Nielsen, \& Faltings, 1991; Gentner \& Stevens, 1983; Reiger \& Grinberg, 1977; Williams, Hollan, \& Stevens, 1983). These studies suggest an important function of dynamic spatial imagery in reasoning and problem solving.

Most of the studies mentioned above are based on subjective reports. How can we obtain objective evidence that reasoning and problem-solving tasks involve spatial visualization processes? In this article, we use the dualtask paradigm (Brooks, 1968) to provide such evidence. That is, we test whether mechanical reasoning impairs a simultaneously held visuospatial working memory load more than it impairs a simultaneously held verbal working memory load.

This research was supported by a National Academy of Education Spencer Fellowship awarded to M.H. We wish to thank Akira Miyake and two anonymous reviewers for comments on an earlier version of this manuscript and Stephanie Thompson, Amy Horowitz, and David Oglesby for research assistance. Correspondence should be addressed to M. Hegarty, Department of Psychology, University of California, Santa Barbara, CA 93106 (e-mail: hegarty@condor.psych.ucsb.edu) or V. K. Sims, Psychology Department, Cedar Crest College, 100 College Drive, Allentown, PA 18104 (e-mail: vksims@ccc-s.cedarcrest.edu).

\section{The Experimental Task}

We examined the effects of visuospatial interference on a task that we refer to as the motion verification task. In this task, people are shown a diagram of a mechanical system and are required to infer the motion of one of the system components on the basis of the spatial configuration of the system and information about how another component is moving. For example, in the sample item shown in Figure 1, people are told to imagine that the free end of the rope is being pulled down and their task is to verify whether the lower pulley will move in the direction shown by the arrow. At least on face value, this task involves the process of mentally simulating the behavior of parts of a device, as would be required to invent, assemble, or troubleshoot a device.

Hegarty and Sims (1994) proposed three stages in the solution of a motion verification item. The first stage is diagram comprehension. At this stage, the solver inspects the diagram and constructs a static spatial representation of the configuration of the mechanical system. The second stage is mental animation. At this stage, the solver infers the motion of the component in question from a given motion (e.g., the motion of the pull rope in a pulley system or the driver in a gear system). The third stage is comparison. At this stage, the solver compares the inferred motion to the motion indicated by an arrow on the component in question to determine whether the answer is true or false.

Hegarty (1992) proposed a piecemeal model of the mental animation stage. According to this model, people decompose the representation of the mechanical system into smaller units, corresponding to the machine components and infer the motion of these components one by one in a sequence corresponding to the causal sequence of events in the machine's operation. For example, to infer the motion of the lower pulley in the item in Figure 1 , a solver first infers that the rope is moving to the 


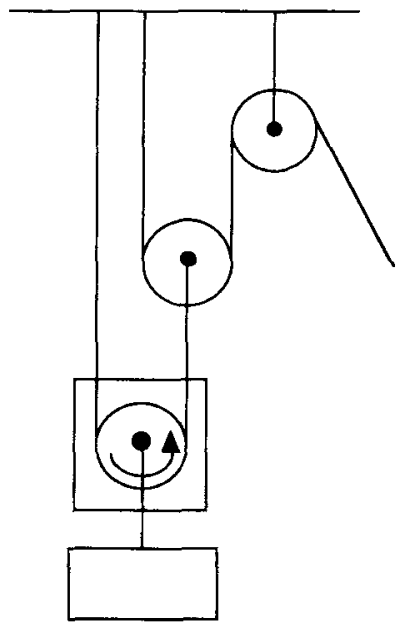

Figure 1. A sample motion verification item.

right over the upper pulley, then infers that the upper pulley is turning clockwise and that the right rope strand is moving up. From this information, he/she goes on to infer that the rope is moving to the right under the lower pulley, which enables him/her to infer that the lower pulley is turning counterclockwise. According to this model, people have to make a longer chain of inferences when inferring the motion of components later in the causal chain of events in the operation of a mechanical system. Consistent with the model, participants in previous experiments made more errors and required more time to infer the motion of these components. Furthermore, eye-fixation data indicated that when asked to infer the motion of a particular component, participants typically looked at that component and earlier components in the causal chain of events, but not at later components (Hegarty, 1992; Hegarty \& Sims, 1994).

\section{Spatial Visualization and Mental Animation}

The model of mental animation proposed by Hegarty (1992) was intended primarily as an account of how a person decomposes the task of inferring the motion of components of a complex mechanical system made up of several interacting components. The model was expressed as a production system. However, it was not meant to imply that the process of inferring the motion of a component of a mechanical system is a rule-based reasoning process. In other words, evidence for a piecemeal mental animation process does not inform us whether individuals infer the motion at each "link" in the causal chain by matching production rules against a propositional representation of a mechanical system (a structural description) or by a spatial visualization process in which they mentally transform an image of one or more components of the mechanical system.

Several recent studies suggest that at least early in practice, mental animation might involve dynamic spatial imagery. Previous research has suggested that spatial visualization ability requires the accurate transformation of visual images (Kosslyn, Brunn, Cave, \& Wallach, 1985; Poltrock \& Agnoli, 1986; Smith, 1964). Therefore, if mental animation involves dynamic spatial imagery, then people who have good spatial visualization ability should perform well on mental animation tasks. In a previous study (Hegarty \& Sims, 1994), we examined the relation of spatial visualization ability to accuracy on the motion verification task. Spatial visualization ability had strong and consistent effects on performance of the motion verification task, whereas verbal ability was unrelated to performance on this task. Although not conclusive, these results suggest that mental animation is a spatial visualization process.

Second, a number of recent studies have argued for a visuospatial inference process on the basis of hand gestures that people make while they are inferring the motion of parts of mechanical systems (Clement, 1994; Hegarty \& Ferguson, 1993; Narayanan, Suwa, \& Motoda, 1994; Schwartz \& Black, 1996b). For example, Schwartz and Black (1996b) have shown that when asked to determine the motions of gears in a gear chain, people begin by enacting the motions of the gears with their hands and formulate rules of mechanical reasoning on the basis of these enactments. In other experimental tasks, such hand movements or enactments have been interpreted as an externalization of spatial imagery transformations (Reisberg \& Logie, 1993).

A third source of evidence for a spatial visualization process comes from studies measuring the time to imagine a displacement of a mechanical component as a function of the size of the displacement. For example, Schwartz and Black (1996a) found that the time to infer the rotation of two interlocking gears was proportional to the angle of rotation of the two gears. This result suggests that inferring gear motion is an analog imagery process.

In this article we use the dual-task paradigm (Brooks, 1968) to argue for the use of spatial visualization processes in mental animation. Selective interference studies offer particularly convincing evidence for the involvement of visual or spatial imagery in task performance. These studies are based on the logic that if a primary task and a concurrent visuospatial secondary task interfere with each other, then visuospatial processes are used in performing the primary task. It must also be shown that the primary task and a concurrent verbal secondary task interfere with each other to a lesser extent.

In the past, dual-task studies have provided evidence for separate verbal and spatial stores in working memory (Baddeley, 1986; Baddeley \& Lieberman, 1980). Specifically, Baddeley has proposed that working memory consists of a central executive and at least two "slave systems" specialized for representing different types of information. These slave systems include the articulatory loop, which is specialized for storing and processing verbal information, and the visuospatial sketchpad, which is specialized for spatial storage and information processing (Baddeley, 1986). Theories of working memory differ regarding how much information processing is attributed to these slave systems. For example, 
Logie (1995) has suggested that the slave systems merely store and rehearse verbal and spatial representations, respectively, and that more complex processing of these representations is carried out by the central executive. In contrast, Shah and Miyake (1996) have argued for separate pools of working memory resources involved in complex spatial information processing (e.g., mental rotation) and verbal information processing (e.g., language comprehension). The present research is based on the assumption that the visuospatial sketchpad is specialized for at least the storage and maintenance of spatial representations. This research also provides information on the locus of more complex spatial information processing.

The dual-task paradigm has also been applied to the study of higher level cognitive processes. For example, Baddeley (1992) used this paradigm to demonstrate that playing chess involves the working memory resources of the visuospatial sketchpad. Similarly, Kruley, Sciama, and Glenberg (1994) used dual-task studies to demonstrate that comprehension of text accompanied by pictures involves the visuospatial sketchpad.

We describe two experiments that examined the role of spatial working memory in motion verification. In Experiment 1 we contrasted the interference effects that occur when motion verification is paired with a visuospatial working memory load to the interference effects that occur when motion verification is paired with a verbal memory load. In Experiment 2, we contrasted the interference effects that occur when visuospatial and verbal memory loads are paired with motion verification to the interference effects that occur when each of these memory loads is paired with a verbal reasoning task that takes approximately the same amount of time as the motion verification task.

\section{EXPERIMENT 1}

In Experiment 1, we examined the degree to which the motion verification task and two different concurrent memory tasks interfere with each other. We will refer to the motion verification as the primary task and to the concurrent memory tasks as secondary tasks. In the secondary tasks, participants were asked to retain in memory either a spatial array of dots or a list of letters. Previous literature (Kruley et al., 1994; Phillips \& Christie, 1977) has suggested that retaining a spatial array of dots uses the working memory resources of the visuospatial sketchpad. In contrast, memory for a list of letters has been shown to use the resources of the articulatory loop (Baddeley \& Hitch, 1974).

We measured the degree to which each concurrent task interferes with performance on the motion verification task and vice versa. If mental animation competes for working memory resources with a memory load, there are several possible outcomes: The memory load will be lost, performance on mental animation will be impaired, or performance on mental animation will be slowed down.
There is also a possibility that time to recall the memory load will be slowed due to mental animation, making it less accessible in memory. Thus, we argue that either decreased accuracy or slower performance on either motion verification or the secondary task can be taken as evidence that the two tasks compete for the same working memory resources. Note that previous studies using the dual-task paradigm have not always examined all possible dependent measures. For example, one task that has been used extensively to measure verbal working memory resources is the reading span task (Daneman \& Carpenter, 1980; Just \& Carpenter, 1992). This task requires subjects to read a group of sentences and recall the final word of each sentence. The measure of reading span is the number of words recalled. That is, it is a measure of accuracy in recalling a memory load, but it does not measure performance on the primary task (sentence comprehension)

Whether impairment occurs on either the primary or secondary task probably depends on the participants' judgments of which task is more important. In a previous dual-task study in which mental rotation was the primary task, Shah and Miyake (1996) found that the interference was evident primarily in loss of the concurrent memory load (i.e., the secondary task). Similarly, in a dual-task study in which comprehension of text accompanied by pictures was the primary task, Kruley et al. (1994) found interference effects only on the secondary task, despite extensive efforts to emphasize accuracy on the secondary task. Thus it is possible that participants will judge any complex spatial task to be more important than a concurrent memory load task and will allocate more resources to that task, in which case we would expect interference effects to be evident primarily in performance on the secondary task.

We hypothesize that mental animation relies on the storage and processing functions of the visuospatial sketchpad. Therefore, we expect to find more impairment when the dot memory task is paired with motion verification than when the letter memory task is paired with mental animation. Notice that we argue for greater interference by a spatial secondary task than by a verbal secondary task, and not for the absence of interference by a verbal secondary task. Because the verbal secondary task was presented visually, it may also require the resources of the visuospatial sketchpad and therefore be interfered with somewhat by the motion verification task. Furthermore, the task-decomposition and goalmanagement components of the motion verification task are most likely to involve the resources of the central executive, so we would expect motion verification to interfere somewhat with any secondary task.

\section{Method}

\section{Participants}

Forty-two students from the University of California, Santa Barbara, took part in this experiment. Two students' data were not included in the analyses because they performed lower than chance 
level on the motion verification task. Of the remaining 40 participants, 20 took part in the verbal memory load condition and 20 took part in the spatial memory load condition. Participants either received course credit or were paid $\$ 5.00$ for their participation.

\section{Design}

This study used a 2 (type of secondary task) $\times 2$ (interference) $\times 3$ (position in causal chain) mixed design. Type of secondary task was manipulated between subjects. In addition to performing motion verification, participants were asked to hold a working memory load that was either verbal or spatial. The presence or absence of a working memory load was manipulated within subjects. Each participant was asked to perform the motion verification task both with and without a memory load as interference. For interference trials, the participant was given a memory load and asked to verify it after performing motion verification. For the no-interference trials, the participant was given a memory load, was asked to verify it, and then performed motion verification. Position in the causal chain was also a within-subjects factor. Participants were given motion verification trials that asked them to verify one of three positions in the causal chain of events: beginning, middle, or end.

Four dependent variables were computed: percent error on the motion verification task, percent error on the secondary task, reaction time (in seconds) on the motion verification task, and reaction time (in seconds) on the secondary task.

\section{Apparatus}

Stimuli were presented on Macintosh IIci computers using MindLab (Meike, Bharucha, Baird, \& Stoeckig, 1988) software. MindLab is a programming shell that presents stimuli and collects responses. It has a temporal resolution of $16.8 \mathrm{msec}$

\section{Materials}

Motion verification task. The motion verification task was used in previous research on mental animation (Hegarty, 1992; Hegarty $\&$ Sims, 1994). It consisted of 24 unique stimuli. Each stimulus showed a diagram of a pulley system drawn in black on a white background. In addition, a red box was drawn around one of the pulleys, and a red arrow was drawn, showing a possible motion of this pulley. The participant's task was to decide if the arrow showed the correct direction of movement if the free end of the rope in the pulley system were pulled. Twelve stimuli showed true movements, and 12 showed false movements. Figure 1 shows a sample stimulus.

Participants were presented with diagrams of two different pulley systems and the mirror images of these systems (producing left and right isomers of the two systems). Each pulley system contained three pulleys. One pulley was at the beginning of the causal chain in the system, the second was in the middle of the causal chain, and the third was at the end of the causal chain. In Figure 1, the upper pulley is toward the beginning of the causal chain, because its motion is caused directly by pulling the free end of the pull rope. In turn, the middle pulley is in the middle of the causal chain because its motion is caused by the movement of the upper rope, which in turn has been caused by pulling on the free end of the rope. Similarly, the lower pulley is toward the end of the chain of events because its movement takes place as a result of several previous movements, including those of the upper and middle pulleys.

Visuospatial secondary task. The visuospatial interference task was adapted from Kruley et al. (1994). On each trial, the participant was first shown a $4 \times 4$ grid containing three dots. His/her task was to memorize the pattern of dots for later verification. The positions of the dots were determined using a random-number generator. The only constraint was that the three dots could not fall in a straight line. The initial presentation of the dot pattern lasted two thirds of a second. For verification, participants were presented with a second grid containing three dots. For half of the trials, this grid was identical to the initial grid (true trial). For the other half of the trials, one randomly chosen dot was moved by one space on the

\section{A. Visuo-Spatial Secondary Task}
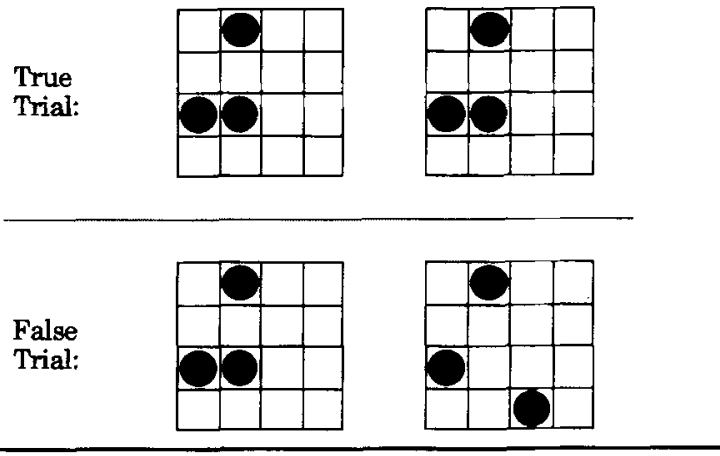

B. Verbal Secondary Tagk

$\begin{array}{ll}\text { True } & \text { LVYQMX }\end{array}$

$\begin{array}{ll}\text { False } & \text { DMKXRG }\end{array}$

Figure 2. Examples of true and false trials for the visuospatial and verbal secondary tasks.

grid (false trial). The direction of movement of this dot was determined using a random-number generator and was constrained in such a way that a straight line was not formed. Examples of true and false trials are shown in Figure 2A.

Verbal secondary task. The verbal secondary task was adapted from Sternberg (1969) and is similar to verbal interference tasks used in other dual-task studies (e.g., Baddeley \& Hitch, 1974). On each trial, a list of six letters appeared for $5 \mathrm{sec}$. Letter lists were constructed using a random-number generator. No letter was repeated within a letter list and no vowels were used, thus minimizing the chances of creating a pronounceable string of letters. For verification, participants were shown a single letter, and they had to decide if this letter was a part of the initial list of six letters. Trials were constructed so that half were true and half were false. Examples of true and false trials are shown in Figure $2 \mathrm{~B}$.

Combination of tasks. The verbal and visuospatial secondary tasks were combined with the motion verification task to produce four different conditions. In the visuospatial memory load condition, participants were presented with a dot grid as a memory load and were then asked to solve a motion verification problem; finally they were asked to verify the initial memory load. For the verbal memory load condition, participants were shown a list of letters, solved a motion verification problem, and then verified a single letter. In the no-load conditions, participants were presented with either a visuospatial or a verbal load, were asked to verify it, and then solved a motion verification problem. True and false motion verification questions were paired with true and false secondary trials, producing equal numbers of the four possible trial types. Additionally, trials were constructed so that each pulley system was paired with the same number of true and false distractor trials. For both of the secondary tasks, as well as the motion verification task, participants responded "true" by pressing the "k" key on the computer keyboard, and "false" by pressing the " $\mathrm{d}$ " key. For ease in responding, a green sticker, labeled "T," was placed on the true key, and a red sticker, labeled "F," on the false key.

\section{Procedure}

Participants were run in groups of $1-5$, and each group was assigned to either the verbal interference or the spatial interference condition. First. participants were given directions and eight prac- 
tice trials for the secondary task. Directions informed them that they would be briefly seeing a series of letters or a collection of dots and that they would later have to verify what they had seen. Examples of both true and false trials were also presented, along with explanations of the correct responses. Next, participants were given directions explaining the motion verification trials. The directions described all of the parts of the pulley systems and explained the motion verification task. Participants then completed eight practice trials for the motion verification task. At this point, participants had a chance to ask questions about either task. Participants were then given the memory load and no-load versions of the main task. The order of these tasks was counterbalanced so that approximately half of the participants received the memory load condition first, and approximately half received the no-load condition first. At the end of the experiment, participants were thanked and dismissed.

\section{Results and Discussion}

We examined selective interference effects on four different dependent measures - accuracy on the primary and secondary tasks and reaction time on the primary and secondary tasks. Using the Dunn-Bonferroni procedure for multiple a priori comparisons, we set the alpha level at $p<.0125$ (Winer, Brown, \& Michels, 1991). All reported effects are significant at this level, unless otherwise noted.

We first compare overall performance in the load and no-load conditions for the motion verification task and the memory load tasks. We later investigate how these effects are modulated by difficulty of the motion verification items (as measured by distance of the component in question from the beginning of the causal chain).

\section{Performance on the Motion Verification Task}

We predicted that the visuospatial secondary task would interfere more with motion verification than would the verbal secondary task. If this is the case, performance on the motion verification task in the presence of a visuospatial working memory load should be poorer

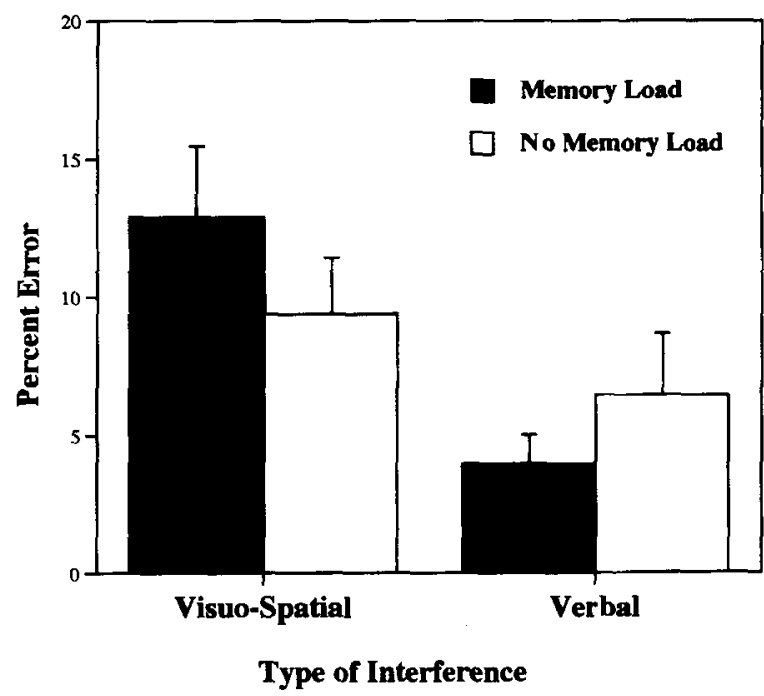

Figure 3. Percent error on the motion verification task in Experiment 1.

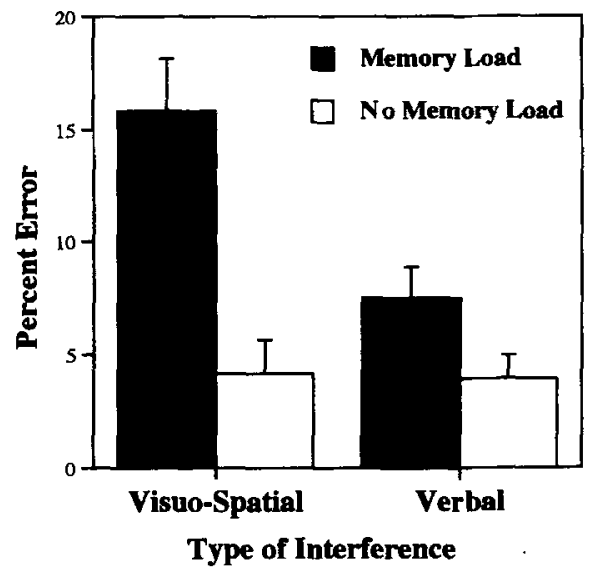

Figure 4. Percent error on the secondary tasks in Experiment 1.

than performance on the motion verification task in the presence of a verbal memory load. We analyzed error on the motion verification task in a 2 (type of secondary task) $\times 2$ (presence of a memory load) mixed analysis of variance (ANOVA). As shown in Figure 3, the interaction of presence of a memory load with type of secondary tasks was marginally significant $[F(1,38)=$ 5.42, $\left.M S_{\mathrm{e}}=33.67, p=.025\right]$. Planned comparisons indicated that when a memory load was present, the spatial interference group made more errors than did the verbal interference group $[t(38)=3.25$, one-tailed]. However, when there was no memory load, the performance of two groups did not significantly differ $[t(38)=$ $0.97]$. These results indicate that motion verification performance was impaired when there was a visuospatial memory load, as would be expected if both tasks were sharing the resources of the visuospatial sketchpad. The results also indicate that the groups were matched on mental animation performance when there was no load. There was also a marginal main effect for motion verification performance to be poorer for the group with the visuospatial secondary task than for the group with the verbal secondary task $\left[F(1,38)=5.33, M S_{\mathrm{e}}=132.36\right.$, $p=.026]$. The main effect for presence of a memory load was not statistically significant.

A separate ANOVA on the reaction times for the motion verification task revealed no significant effects of either type of interference task or presence of a memory load on this measure.

\section{Performance on the Secondary Tasks}

First, we established that the two secondary tasks were of equivalent difficulty when a motion verification trial did not intervene between presentation and verification of the memory load. In the no-load conditions, the error rate for the dot memory task was low (4.17\%) and was not significantly different from the error rate for the letter memory task, which was $3.96 \%[t(38)=0.12]$. Figure 4 shows the means and standard errors for error rate on the secondary tasks in the load and no-load conditions. 


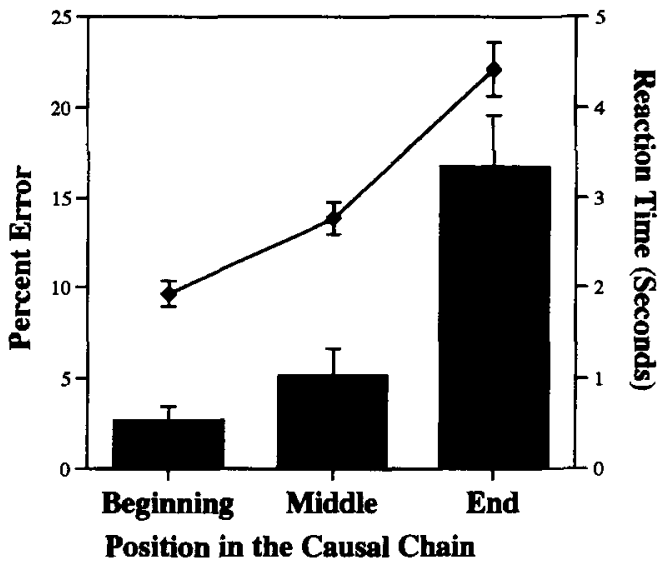

Figure 5. Percent error and reaction time on the motion verification task as a function of position in the causal chain in Experiment 1 . The bars show the percent error, and the line shows the reaction time.

We predicted that memory for the secondary task would be poorer in the load conditions than in the noload conditions and that this decrement in performance would be greater for the visuospatial task (dot memory) than for the verbal task (letter memory). A 2 (type of secondary task) $\times 2$ (presence of a memory load) mixed ANOVA revealed a significant main effect of presence of a memory load $\left[F(1,38)=33.36, M S_{\mathrm{e}}=34.67\right]$. More critically, presence of a memory load interacted with type of secondary task $\left[F(1,38)=9.52, M S_{\mathrm{e}}=34.67\right]$. As predicted, there was a greater decrement in performance on memory for dot configuration (an accuracy difference of $11.66 \% ; S D=9.99)$ than on memory for letters $(3.54 \%$; $S D=6.25$ ). Again, this is consistent with the view that mental animation and dot memory compete for the resources of the visuospatial sketchpad. The main effect for a higher error rate by the group with the visuospatial memory task also approached statistical significance $\left[F(1,38)=5.2, M S_{\mathrm{e}}=70.12, p=.02\right]$.

A comparison of reaction times in the no-load conditions indicated that it took longer to verify a letter $(M=$ $1.44 \mathrm{sec}, S D=.32)$ than a dot pattern $[M=.96 \mathrm{sec}$, $S D=.24, t(38)=5.38]$. Therefore, we standardized the reaction time data by converting the data for each task to $z$ scores. These $z$ scores were then subjected to a 2 (type of interference task) $\times 2$ (presence of a memory load) mixed ANOVA. Participants were slower on the secondary task when a motion verification trial intervened between presentation and verification of the memory load $(M=.46, S D=1.11)$ than when a motion verification trial did not intervene $[M=-.44, S D=.59$, $\left.F(1,38)=37.58, M S_{\mathrm{e}}=.43\right]$. Reaction time was not affected significantly by type of secondary task or the interaction of type of secondary task with presence of a memory load.

\section{Effects of Position in the Causal Chain on Performance}

As in previous research (Hegarty, 1992; Hegarty \& Sims, 1994), participants made more errors in verifying the motion of components later in the causal chain [see Figure 5; $F(2,78)=20.62, M S_{\mathrm{e}}=109.21$ ]. Reaction time to verify the motion of a pulley also increased monotonically with its distance from the beginning of the causal chain of events $\left[F(2,78)=76.10, M S_{\mathrm{e}}=.847\right]$. Therefore, items about components later in the causal chain are more difficult and require more time. According to the piecemeal model of mental animation (Hegarty, 1992), this is because components are mentally animated one by one in the order of the causal chain of events.

To investigate how difficulty and processing time of a motion verification item affect loss of a concurrent memory load, we analyzed the effects of position in the causal chain on errors and reaction time on the secondary task. This analysis was based on difference scores between the load and no-load conditions (i.e., the loss of a memory load when motion verification intervenes between storage and recall minus the loss of memory load when there is no intervening task). These difference scores are shown in Figure 6. The difference scores were then subjected to a 3 (position in the causal chain) $\times 2$ (type of secondary task) ANOVA. In the analysis based on errors, the effect of position in the causal chain did not reach significance $\left[F(2,76)=1.99, M S_{\mathrm{e}}=135.83\right]$, nor did its interaction with type of interference task $\left[F(2,76)=2.19, M S_{\mathrm{e}}=\right.$ 135.83].

In the analysis based on reaction time, there was a significant effect of position in the causal chain $[F(2,76)=$ $\left.5.31, M S_{\mathrm{e}}=.21\right]$, indicating that verification of both memory loads was slowed down as a function of the time taken for the intervening motion verification item. Position in the causal chain did not interact with type of secondary task.

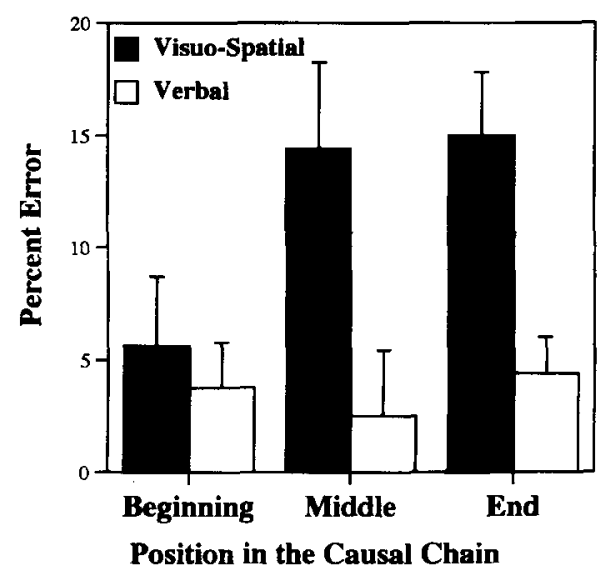

Figure 6. Difference in percent error on the secondary task between the load and no-load conditions in Experiment 1. 


\section{EXPERIMENT 2}

Experiment 1 established that mental animation interferes more with memory for the spatial configuration of dots than with memory for a sequence of letters. It also suggested that the dot memory task interferes more with mental animation than does the letter memory task. These results are highly suggestive that mental animation uses the resources of the visuospatial sketchpad. However, we need to address the alternative hypothesis that holding a dot configuration in memory consumes more working memory resources in general than does holding a sequence of letters in memory. If this is the case, we should observe greater interference when dot memory is paired with any task, and not just when it is paired with a spatial visualization task. In order to rule out this alternative hypothesis, it is necessary to establish that there is a task with which dot memory does not interfere as much as does letter memory.

In Experiment 2, we examined the degree to which the dot memory task and the letter memory task interfere with two different primary tasks: motion verification and a verbal reasoning task. The verbal reasoning task was adapted from Baddeley and Hitch (1974) and was chosen because pilot studies established that it takes approximately the same amount of time as the motion verification task. In this task, the participant is asked to verify the order of two letters of the alphabet. Researchers have argued that this task primarily requires the resources of the central executive, with some dependence on the articulatory loop, but that it does not use the resources of the visuospatial sketchpad. Thus, performance on this task is impaired somewhat by articulatory suppression, but not at all by spatial tapping (Farmer, Berman, \& Fletcher, 1986; Hitch \& Baddeley, 1976). We predict an interaction between type of memory load and type of main task (motion verification or verbal reasoning task). First, participants should show greater impairment when the dot memory task is paired with motion verification than when it is paired with the verbal reasoning task. Second, participants should show more impairment when the letter memory task is paired with the verbal reasoning task than when the letter memory task is paired with motion verification.

\section{Method}

\section{Participants}

Forty-six students from the University of California, Santa Barbara, received course credit to take part in Experiment 2. Six of these participants were deleted from the final analysis because their performance on the verbal reasoning task was poorer than chance. Subsequent analysis of their answers revealed that they had answered fewer than a quarter of the passive items correctly, suggesting that they had misunderstood the instructions for the verbal reasoning task. Participants were assigned to one of two groups: visuospatial interference or verbal interference. The final sample contained 20 participants in the visuospatial interference group, and 20 participants in the verbal interference group.

\section{Design}

This study used a 2 (type of reasoning task) $\times 2$ (type of memory interference) mixed design. Type of reasoning task was manipulated within subjects. Each participant performed two primary tasks: the Baddeley and Hitch (1974) verbal reasoning task and motion verification. Type of interference was manipulated between subjects. Participants completed both main tasks while carrying either a verbal memory load (letters) or a spatial memory load (dot configuration).

As in Experiment 1, the motion verification task contained trials asking about the beginning, middle, and end of the causal chain. The verbal reasoning task required that participants verify both active and passive statements. We measured the same four dependent variables as those in Experiment 1.

\section{Apparatus}

As in Experiment 1, stimuli were presented on Macintosh IIci computers using Mindlab (Meike et al., 1988) software.

\section{Materials}

In this experiment, participants completed a verbal reasoning task and a motion verification task, each while performing a secondary task. The motion verification task and both of the secondary interference tasks (dot memory and letter memory) were identical to those used in Experiment 1.

Verbal reasoning task. The verbal reasoning task used in this experiment was adapted from Baddeley and Hitch (1974). On each trial, a sentence appeared followed by two letters. Each sentence described the ordering of those letters; for example:

\section{A precedes $B \quad A B$}

The letters presented after the sentence were always in alphabetic order. The participant's task was to decide whether the sentence ac curately described the order of the letters. Twenty-four trials were constructed in the following manner. Sentences were written either in the active voice (as in the above example) or the passive voice (e.g., "A is preceded by B AB.") and used either the verb precedes or the verb follows. Twelve sentences asked about the ordering of the letters $a$ and $b, 12$ about the ordering of $c$ and $d$, and 12 about the ordering of $e$ and $f$. Half of the trials were true, and half were false. As in the motion verification condition, the secondary verbal and visuospatial tasks were paired with the verbal reasoning task so that equal numbers of true and false distractor trials were paired with active and passive statements in the verbal reasoning task.

Combination of tasks. The verbal and visuospatial secondary tasks were combined with motion verification and verbal reasoning to produce four different conditions. In the visuospatial memory load conditions, participants were presented with a dot grid as a memory load, then solved a motion verification problem or a verbal reasoning problem, and finally verified the initial memory load. In the verbal memory load conditions, participants were shown a list of letters, solved a motion verification problem or a verbal reasoning problem, and then verified that a single letter was a part of the memory load. As in Experiment 1, true and false motion verification questions were paired with true and false secondary trials, producing equal numbers of the four possible trial types. Again, participants responded by pressing " $k$ " for true and "d" for false.

\section{Procedure}

Participants were run in groups of $1-5$, and all performed both motion verification and verbal reasoning as primary tasks. Half of the participants performed these tasks with a visuospatial memory load, and half performed these tasks with a verbal memory load. The order of tasks was counterbalanced so that approximately half of the participants received the motion verification task first and 
approximately half received the verbal reasoning task first. As in Experiment 1, participants received instructions and eight practice trials for each experimental task before they performed that task. In each condition of the experiment, participants received two replications of the stimuli.

\section{Results}

The data were analyzed separately for error on the primary tasks, reaction time on the primary tasks, error on the secondary tasks, and reaction time on the secondary tasks. The alpha level was again set at $p<.0125$. We first compare performance on the primary tasks (verbal reasoning and motion verification) and then examine how these tasks affected verification of the memory load (either visuospatial or verbal). We later investigate how these effects were modulated by difficulty of the motion verification items (position in the causal chain) and the verbal reasoning items (verification of active or passive sentences).

\section{Performance on the Primary Tasks}

We analyzed performance on the primary tasks in two 2 (type of primary task) $\times 2$ (type of secondary task) mixed ANOVAS, with errors and reaction times as the dependent variables. Performance on the motion verification and verbal reasoning tasks revealed that as in our pilot testing, the mean time taken for a motion verification trial ( $3.22 \mathrm{sec}, S D=1.19$ ) was approximately equal to the mean time taken for a verbal reasoning item $[3.46 \mathrm{sec}$, $\left.S D=1.11, F(1,38)=1.09 . M S_{\mathrm{e}}=1.06\right]$. However, there was a nonsignificant trend for participants to make more errors on the verbal reasoning task $(12.87 \%, S D=13.1)$ than on the motion verification task $[8.18 \%, S D=8.29$, $\left.F(1,38)=3.75, M S_{\mathrm{e}}=117.13, p=.06\right]$.

A possible account of the results of Experiment 1 is that holding a dot configuration in memory consumes more working memory resources in general than does holding a sequence of letters in memory. If this is the case, performance on both the motion verification and verbal reasoning tasks should be poorer when they are paired with dot memory than when they are paired with letter memory. However, in Experiment 2, performance on the primary tasks was not significantly affected by the type of secondary tasks (dot memory or letter memory) $\left[F(1,38)=.55, M S_{\mathrm{e}}=128.46\right.$ for error rate; $F(1,38)=$ $1.10, M S_{\mathrm{e}}=1.61$ for reaction time].

This experiment did not replicate the trend in Experiment 1 for mental animation performance to be more impaired by a visuospatial working memory load than by a verbal working memory load. There were no significant interactions of type of primary task with type of secondary task $[F(1,38)<1$ for both error rate and reaction time]. The results were in the same direction as those in Experiment 1. Participants receiving the visuospatial interference task made more errors on the motion verification task $(9.2 \%)$ than did participants receiving the verbal memory load (7.1\%). However, these participants also made more errors on the verbal reasoning task
$(13.6 \%)$ than did participants who received the verbal memory load $(12.1 \%)$.

\section{Performance on the Secondary Tasks}

We predicted that participants would show greater impairment on dot memory when it was paired with the motion verification task than when it was paired with the verbal reasoning task and that participants should show more impairment on the letter memory task when it was paired with the verbal reasoning task than when it was paired with the motion verification task. We analyzed errors on the secondary task in a 2 (type of primary task) $\times 2$ (type of secondary task) ANOVA. As shown in Figure 7, there was a significant interaction between type of primary task and type of secondary task $[F(1,38)=$ $\left.20.54, M S_{\mathrm{e}}=61.01\right]$. As predicted, participants were more impaired on dot memory when they performed an intervening motion verification item and were more impaired on letter memory when they performed an intervening verbal reasoning item. As in Experiment 1, planned comparisons indicated that mental animation interfered more with the visuospatial secondary task than with the verbal secondary task $[t(38)=4.05]$. However, verbal reasoning did not interfere significantly more with the verbal secondary task than with the visuospatial secondary task. Furthermore, dot memory was more impaired by motion verification than by verbal reasoning $[t(19)=$ 3.39] and letter memory was more impaired by verbal reasoning task than by motion verification $[t(19)=3.15]$. These results provide strong evidence that mental animation and dot memory share working memory resources (i.e., the visuospatial sketchpad) that are distinct from those used by verbal reasoning and letter memory.

Since participants were slower to verify letters $(M=$ $1.81 \mathrm{sec}, S D=.32 \mathrm{sec})$ than dot configurations $(M=$ $1.37 \mathrm{sec}, S D=.23 \mathrm{sec}$ ), as in Experiment 1, the reaction time data were converted to $z$ scores. We then analyzed the standardized data in a 2 (type of primary task) $\times 2$ (type of secondary task) ANOVA. The interaction of

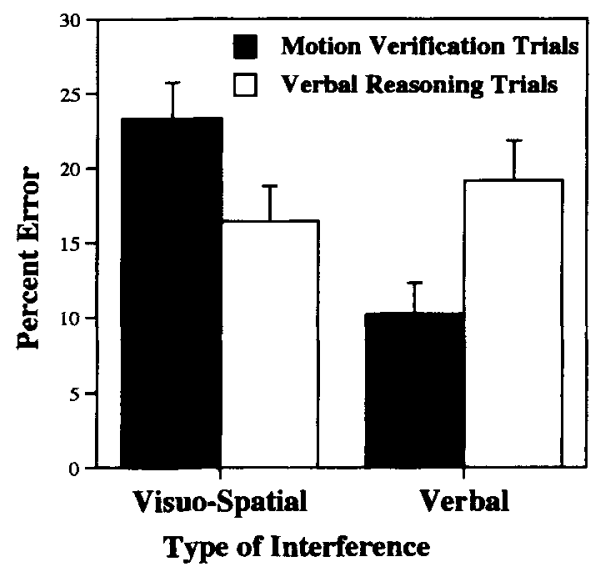

Figure 7. Percent error on the secondary tasks in Experiment 2. 
type of primary task with type of secondary task was not statistically significant $\left[F(1,38)=2.21, M S_{\mathrm{e}}=.63\right]$, nor was the main effect of type of secondary task $[F(1,38)=$ 1.37]. Although the primary tasks slowed down the verification of the memory loads in the predicted direction, the significant interference effects in Experiment 2 were in loss of the memory loads.

\section{Effects of Position in the Causal Chain on Performance}

For the motion verification trials, error and reaction time data were analyzed in a 3 (position in the causal chain) $\times 2$ (type of secondary task) ANOVA. As in previous research (Hegarty, 1992; Hegarty \& Sims, 1994) and in Experiment 1, participants made more errors in verifying the motion of components later in the causal chain [see Figure $8 ; F(2,76)=21.83, M S_{\mathrm{e}}=127.12$ ]. Reaction time to verify the motion of a pulley also increased monotonically with its distance from the beginning of the causal chain of events [see Figure $8 ; F(2,76)=$ $\left.62.71, M S_{\mathrm{e}}=1.42\right]$. Position in the causal chain did not interact with type of secondary task for the measures of performance on the motion verification task.

To investigate how difficulty and processing time of a motion verification item affect loss of a concurrent memory load, we analyzed the effects of position in the causal chain on errors and reaction time for the secondary task in a 3 (position in the causal chain) $\times 2$ (type of secondary task) ANOVA. As shown in Figure 9 , position in the causal chain interacted with type of secondary task $\left[F(2,76)=15.87, M S_{\mathrm{e}}=56.54\right]$. Simple effects indicated that position in the causal chain affected memory for a dot pattern $\left[F(2,76)=22.26, M S_{\mathrm{e}}=56.54\right]$ more than it affected memory for a list of letters $[F(2,76)$ $\left.=2.84, M S_{\mathrm{e}}=56.54\right]$. Curiously, motion verification items about the middle pulley in the causal chain caused more loss of the visuospatial working memory load than did items about the beginning or end pulleys. A tentative

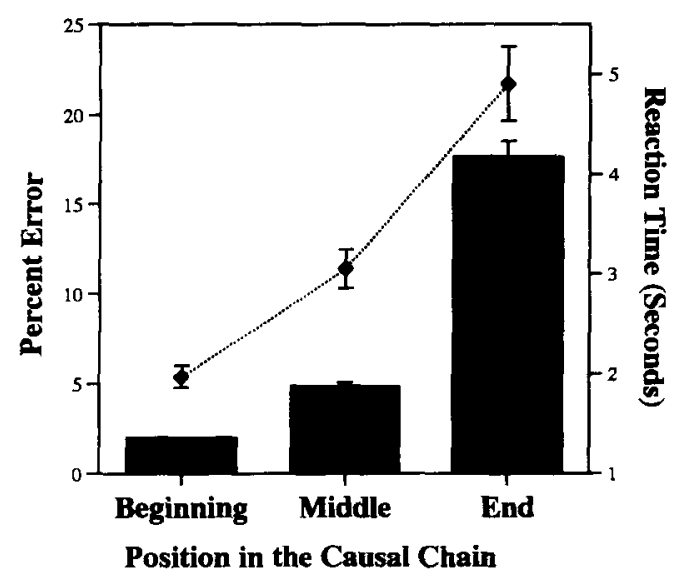

Figure 8. Percent error and reaction time on the motion veriffcation task as a function of position in the causal chain in Experiment 2 . The bars show the percent error, and the line shows the reaction time.

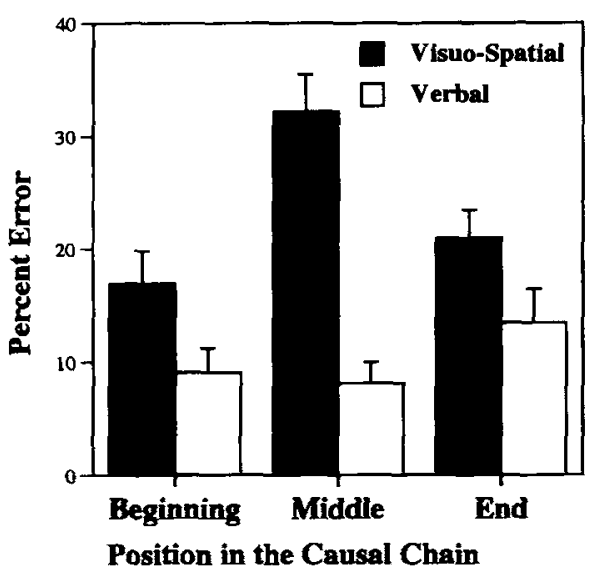

Figure 9. Percent error on the distractor task in Experiment 2 as a function of position in the causal chain.

explanation of this effect is that the middle pulley in both pulley systems has more connections to other components in the system, making it more visually complex. Therefore, more working memory resources might be required to represent this pulley. However, this effect might also be an artifact due to more difficult distractor trials being paired with items about the middle pulley (all participants received the same pairings of distractors with motion verification items).

In the analysis of reaction times for the secondary task, there was also a marginal interaction of type of secondary task with position in the causal chain $[F(2,76)=$ 3.32, $\left.M S_{\mathrm{e}}=.714, p=.04\right]$. Simple effects analyses indicated a marginal effect of position in the causal chain for the visuospatial secondary task $[F(2,76)=4.16$, $\left.M S_{\mathrm{e}}=.714, p=.02\right]$. As Figure 10 shows, reaction time on the visuospatial secondary task increased with distance of a component from the beginning of the causal chain. In contrast, there was no significant effect of position in the causal chain on reaction time for the verbal secondary task $(F<1)$. This result is consistent with the interpretation that mental animation and dot memory share the resources of the visuospatial sketchpad-if a trial involves more mental animation steps, it renders the dot pattern less accessible in working memory. An alternative explanation is that the increased time to verify a dot pattern is merely a function of the amount of time intervening between first seeing the pattern and later verifying it. However, note that this effect does not occur for the verbal memory load.

\section{Effects of Voice (Passive or Active) in the Verbal Reasoning Task}

As in previous research (Baddeley \& Hitch, 1974), there was a trend for participants to make more errors verifying passive sentences $(16.04 \%, S D=20.1)$ than active sentences $\left[9.69 \%, S D=10.77, F(1,38)=4.78, M S_{\mathrm{e}}=\right.$ $169.02, p<.05]$. They also took more time to verify passive sentences $(3.81 \mathrm{sec}, S D=1.36)$ than active sentences $\left[3.18 \mathrm{sec}, S D=.97, F(1,38)=36.06, M S_{\mathrm{e}}=.22\right]$. How- 


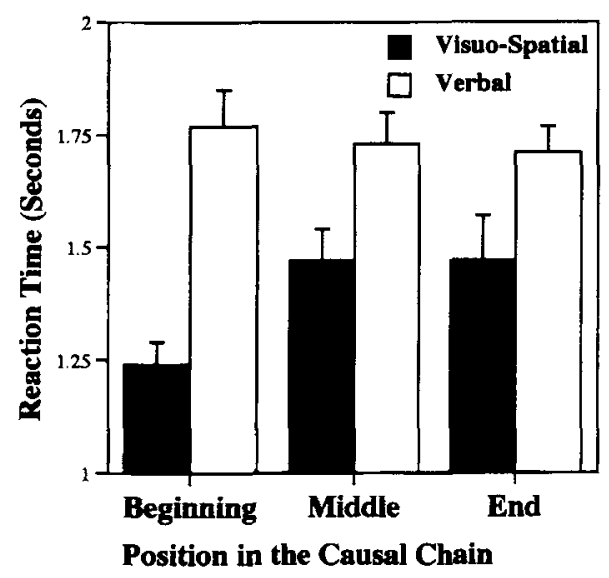

Figure 10. Reaction time on the distractor task in Experiment 2 as a function of position in the causal chain.

ever, the difficulty of a verbal reasoning task (as measured by voice) affected neither the accuracy nor the time to verify a memory load ( $F<1$ for both measures). Difficulty also did not interact with type of secondary task. Note that passive sentences did not increase the reaction time to verify a dot pattern. Thus the observed effects of position in the causal chain on reaction time to verify a dot pattern can be interpreted as selective interference effects and not merely decay with time required by the intervening (primary) task.

\section{GENERAL DISCUSSION}

In this research, we demonstrated that mental animation of a pulley system interferes more with memory for a concurrent visuospatial array than with memory for a list of letters. Experiment 1 also showed that a visuospatial working memory load interferes more with mental animation than does a verbal memory load. Furthermore, Experiment 2 indicated that mental animation interferes more with a visuospatial memory load than does a verbal reasoning task that takes approximately the same amount of time. These results argue for a dissociation between the working memory resources required by mental animation and those required by verbal reasoning and suggest that mental animation is dependent on the resources of the visuospatial sketchpad.

The evidence presented in this article is consistent with the model proposed by Baddeley (1986), who suggested that working memory contains separate "slave systems" for storing and maintaining visuospatial and verbal information. Our results also add to a growing body of evidence that the visuospatial sketchpad is involved in high-level comprehension and reasoning tasks that involve spatial representations (Baddeley, 1992; Kruley et al., 1994). In fact, the visuospatial sketchpad has been proposed as the site of mental models. Kruley et al. have suggested that when people read a text accompanied by a picture (e.g., a description of a spatial environment), they construct a mental model of the ref- erent in the visuospatial sketchpad. In these situations, "mental model" refers to a spatial representation of the referent of the text and/or picture. In research on reasoning about physical systems, the term "mental model" has been used to refer to a dynamic spatial representation that allows one to mentally simulate the operation of a system (DeKleer \& Brown, 1984; Forbus et al., 1991; Gentner \& Stevens, 1983; Reiger \& Grinberg, 1977; Williams et al., 1983). Our research provides evidence that this type of dynamic mental model also requires the resources of the visuospatial sketchpad.

The study of dynamic spatial reasoning tasks such as mental animation can provide important information about the structure of working memory. For example, a point of current controversy is whether the visuospatial sketchpad has specialized resources for both storage and processing of spatial information or whether it is merely a static store for spatial information, with spatial processing being carried out by the central executive (Logie, 1995; Shah \& Miyake, 1996). It would be interesting to compare the memory load requirements of mental animation and a task that merely requires inspecting a static representation. For example, Hegarty (1992) compared the motion verification task used in this research with a static task in which subjects had to verify statements about the spatial relations between components in a pulley system diagram. If the visuospatial sketchpad is specialized for both storage and processing, dynamic mental models should require more spatial working memory resources than do static spatial representations.

The present experiments provided preliminary evidence in support of the view that there are working memory resources specialized for dynamic spatial processing. First, our research showed a dissociation between a dynamic spatial reasoning task and a verbal reasoning task that has been shown to be highly dependent on the central executive (Farmer et al., 1986; Hitch \& Baddeley, 1976). Second, more dynamic tasks (i.e., motion verification trials that involved more spatial transformations) tended to interfere more with memory for a stored spatial array.

In the dual-task paradigm, impairment on either the primary or the secondary task is evidence that two tasks share the same resources. Although there was evidence for impairment in both of our experiments, there was some inconsistency between experiments as to which task was impaired. In both experiments, the secondary task was impaired. This finding is in line with other dual-task studies in which the primary task was a relatively complex spatial task (Kruley et al., 1994; Shah \& Miyake, 1996). In Experiment 1, the primary task also tended to be impaired, but this trend was not replicated in Experiment 2. Although the subjects in the two experiments were given the same instructions, a comparison across experiments suggests that they weighted the tasks differently. When dot memory was paired with mental animation, the mean error on the dot memory task was lower in Experiment $1(15.8 \%)$ than in Experiment $2(23.3 \%)$, and the mean error for mental anima- 
tion was higher in Experiment $1(12.9 \%)$ than in Experiment $2(9.3 \%)$. Therefore, subjects in Experiment 1 seem to have allocated relatively more resources to the secondary task. Further research is required to study the influences on the relative weighting given to the two tasks in a dual-task study.

In this experiment, we used mental animation of pulley systems as an example of a dynamic mechanical reasoning task. Other research has shown that like pulley systems, gear, lever, and more complex systems are also animated piecemeal, and that ability to mentally animate these systems is related to spatial visualization ability (Hegarty \& Steinhoff, 1994). Therefore, we would expect the selective interference results observed here to generalize to the mental simulation of other mechanical devices.

Finally, we might ask whether inferring the motion of a mechanical device from a static diagram (mental animation) is a mental imagery process. Several sources of evidence converge on this view. First, mental animation draws on the resources of the visuospatial sketchpad, which has been characterized as being specialized for the storage and processing of mental images (Logie \& Baddeley, 1990; Riesberg \& Logie, 1993). Second, we have provided evidence elsewhere (Hegarty \& Sims, 1994) that mental animation performance is correlated with spatial visualization ability, which in turn has been characterized as involving the storage and processing of mental images (Kosslyn et al., 1985; Poltrock \& Agnoli, 1986; Smith, 1964). Third, Schwartz and Black (1996a) found that the time to mentally simulate the rotation of two gears is proportional to the angle of rotation, suggesting an analog imagery process. Finally, when people reason about dynamic physical systems, they frequently make hand gestures that simulate the behavior of the different device components (Clement, 1994; Hegarty \& Ferguson, 1993; Narayanan et al., 1994; Schwartz \& Black, 1996b). These gestures are seen as an externalization of internal imagery processes. These different sources of evidence point to a central role of dynamic spatial imagery in mechanical reasoning.

\section{REFERENCES}

BADDELEY, A. D. (1986). Working memory. Oxford: Oxford University Press.

BADDELEY, A. D. (1992). Is working memory working? The fifteenth Bartlett lecture. Quarterly Journal of Experimental Psychology, 44, $1-31$.

Baddeley, A. D., \& Hitch, G. (1974). Working memory. In G. H Bower (Ed.), The psychology of learning and motivation (pp. 47-89). San Diego: Academic Press.

Baddeley, A. D., \& Lieberman, K. (1980). Spatial working memory. In R. Nickerson (Ed.), Attention and performance XVIII (pp. 521539). San Diego: Academic Press.

Brooks, L. R. (1968). Spatial and verbal components of the act of recall. Canadian Journal of Psychology, 22, 349-368.

Clement, J. (1994). Imagistic simulation and physical intuition in expert problem solving. Proceedings of the Annual Conference of the Cognitive Science Society, 16, 201-206.

DANEMAN, M., \& CARPENTER, P. A. (1980). Individual differences in working memory and reading. Journal of Verbal Learning \& Verbal Behavior, 19, 450-466.
DeKleER, J., \& Brown, J. S. (1984). A qualitative physics based on confluences. Artificial Intelligence, 24, 7-83.

Farmer, E. W., Berman, J. V. F., \& Fletcher, Y. L. (1986). Evidence for a visuo-spatial scratchpad in working memory. Quarterly Journal of Experimental Psychology, 38A, 675-688.

Ferguson, E. L., \& Hegarty, M. (1994). Properties of cognitive maps constructed from text. Memory \& Cognition, 22, 455-473.

Ferguson, E. S. (1977). The mind's eye: Nonverbal thought in technology. Science, 197, 827-836.

Forbus, K. D., Nielsen, P., \& Faltings, B. (1991). Qualitative spatial reasoning-The clock project. Artificial Intelligence, 51, 417-471.

Gentner, D., \& Stevens, A. L. (Eds.) (1983). Mental models. Hillsdale, $\mathrm{NJ}$; Erlbaum.

HegarTY, M. (1992). Mental animation: Inferring motion from static displays of mechanical systems. Journal of Experimental Psychology: Learning, Memory, \& Cognition, 18, 1084-1 102.

Hegarty, M., \& Ferguson, J. M. (1993, November). Strategy change - with practice in a mental animation task. Paper presented at the annual meeting of the Psychonomic Society, Washington, DC.

HegarTy, M., \& Sims, V. K. (1994). Individual differences in mental animation during mechanical reasoning. Memory \& Cognition, 22, 411430.

Hegarty, M. \& Steinhoff, K. (1997).Individual differences in use of diagrams as external memory in mechanical reasoning. Learning \& Individual Differences, 9, 19-42.

Hitch, G. J., \& BAdDELEY, A. D. (1976). Verbal reasoning and working memory. Quarterly Journal of Experimental Psychology, 28, 603621

JUST, M. A., \& CARPENTER, P. A. (1992). A capacity theory of comprehension: Individual differences in working memory. Psychological Review, 99, 122-149.

Kosslyn, S. M., Brunn, J., Cave, K. R., \& Wallach, R. W. (1985). Individual differences in mental imagery: A computational analysis. In S. Pinker (Ed.), Visual cognition (pp. 195-243). Cambridge, MA: MIT Press.

Kruley, P., Sciama, S. C., \& Glenberg, A. M. (1994). On-line processing of textual illustrations in the visuospatial sketchpad: Evidence from dual-task studies. Memory \& Cognition 22, 261-272.

LoGIE, R. H. (1995). Visuo-spatial working memory. Hove, U.K.: Erlbaum.

Logie, R. H., \& BADDELEy, A. D. (1990). Imagery and working memory. In P. J. Hampson, D. F. Marks, \& J. T. E. Richardson (Eds.), Imagery: Current developments (pp. 103-J28). London: Routledge.

Meike, B., Bharucha, J. J., Baird, J. C., \& Stoeckig, K. (1988). MindLab. Santa Barbara, CA: Intellimation.

MILLER, A. I. (1984). Imagery in scientific thought. Boston: Birkhauser Narayanan, N. H. , Suwa, M., \& Motoda, H. (1994). A study of diagrammatic reasoning from verbal and gestural data. Proceedings of the Annual Meeting of the Cognitive Science Society, 16, 652657.

NERSESSIAN, N. (1992). How do scientists think? Capturing the dynamics of conceptual change in science. In R. Giere (Ed.), Cognitive models of science (pp. 3-44). Minneapolis: University of Minnesota Press.

O'NeILl, J. J. (1944). Prodigal genius: The life of Nikola Tesla. New York: Ives Washburn.

Phillips, W. A., \& Christie, D. F. M. (1977). Interference with visualization. Quarterly Journal of Experimental Psychology, 29, 637650.

Poltrock, S. E., \& AGNOLI, F. (1986). Are spatial visualization ability and visual imagery ability equivalent? In R. J. Sternberg (Ed.), $A d-$ vances in the psychology of human intelligence (pp. 255-296). Hillsdale, $\mathrm{NJ}$ : Erlbaum.

REIGER, C., \& GRINBERG, M. (1977). The declarative representation and procedural simulation of causality in physical mechanisms. Proceedings of the International Conference on Artificial Intelligence, 5 , 250-255.

Riesberg, D., \& Logie, R. (1993). The ins and outs of working memory: Overcoming the limits on learning from imagery. In B. RoskosEwoldson, M. J. Intons-Peterson, \& R. E. Anderson (Eds.), Imager creativity, and discovery (pp. 39-70). Amsterdam: North-Holland 
SChwarTZ, D. L., \& Black, J. B. (1996a). Analog imagery in mental model reasoning: Depictive models. Cognitive Psychology. 30, 154 219.

Schwartz, D. L., \& BLACK, J. B. (1996b). Shuttling between depictive models and abstract rules: Induction and fall-back. Cognitive Science, 20, 457-497.

SHAH, P., \& MiYAKE, A. (1996). The separability of working memory resources for spatial thinking and language processing: An individ ual differences approach. Journal of Experimental Psychology: General, 125, 4-27.

SHEPARD, R. N. (1978). Externalization of mental images and the act of creation. In B. S. Randhava \& W. E. Coffman (Eds.), Visual learning, thinking, and communication (pp. 133-189). San Diego: Academic Press.
Sмгтн, I. M. (1964). Spatial ability: Its educational and social significance. London: University of London Press.

STERNBERG, S. (1969). Memory scanning: Mental processes revealed by reaction time experiments. American Scientist, 57, 421-457.

Williams, M. D., Hollan, J, D., \& Stevens, A. L. (1983). Human reasoning about a simple physical system. In D. Gentner \& A. L. Stevens (Eds.), Mental models (pp. 131-153). Hillsdale, NJ: Erlbaum.

Winer, B. J., Brown, D. R., \& MichaELS, K. M. (1991). Statistical procedures in experimental design (3rd ed). New York: McGraw-Hill.

(Manuscript received November 14, 1994; revision accepted for publication September 22, 1995.) 